\title{
Fracture Surfaces of Porous Materials
}

\author{
T. Ficker
}

\begin{abstract}
A three-dimensional absolute profile parameter was used to characterize the height irregularities of the fracture surfaces of cement pastes. The dependence of these irregularities on porosity was studied and its non-linear character was proved. An analytical form for the detected non-linearity was suggested and then experimentally tested. The surface irregularities manifest scale-invariance properties.
\end{abstract}

Keywords: roughness analysis, fracture surfaces, cement-based materials, confocal microscopy.

\section{Introduction}

The morphology of fracture surfaces have been studied for a long time to reveal the details of fracture processes. However, in the research field of cementitious materials there have been only a restricted number of studies that deal with surface features of fractured specimens. Some of the early surface studies of hydrated cement materials were focused on fractal properties $[1,2]$ whereas others [3-5] investigated roughness numbers $(R N)$ or similar surface characteristics [6-9].

When dealing with the roughness of the fracture surfaces of porous materials like cementitious materials, an important question arises, namely, what type of relationship is there between surface roughness and porosity. Recently, Ponson at al $[10,11]$ have pointed to a close relationship between these two quantities. The authors studied the roughness of fracture surfaces with glass ceramics made of small glass beads sintered in bulk with porosity $P$ that could be varied in the interval $(0,0.3)$. They observed that the roughness of the fracture surfaces increases linearly with increasing porosity (see their figure 3.3 in [10]). It would be valuable to know whether such a linear behavior is a general property of all porous materials, or only a specific feature of glass ceramics. For this reason we performed a large series of experiments with cement pastes. This material was chosen because its porosity can be easily controlled within a broad porosity interval by means of the water-tocement ratio $r=w / c$. Correct knowledge of the functional dependence of roughness on porosity may be useful for further surface studies of fractured porous materials.

\section{Experimental arrangement}

Ordinary Portland cement CEM 42,5 I R-sc of domestic provenance was used to create 108 specimens of hydrated pastes with six different water-to-cement ratios $r(0.3,0.4,0.5,0.6,0.7,0.8)$. The specimens were rotated during hydration to achieve better homogeneity. All specimens were stored for the whole time of hydration at $100 \% \mathrm{RH}$ and $20^{\circ} \mathrm{C}$. After 60 days of hydration the specimens were fractured in three-point bending tests and the fracture surfaces were immediately used for microscopic analysis. Other parts of the specimens were used for porosity measurements and for further mechanical tests.

Porosity was determined by the common weightvolume method. The wet specimens were weighed and their volume was measured, then they were subjected to $105{ }^{\circ} \mathrm{C}$ for one week until their weight no longer changed, and the dry specimens were weighed again.

The 3D profile parameter $H_{a}$ was used to characterize the roughness of the fracture surfaces of the hydrated cement pastes. In fact, $H_{a}$ represents the averaged 'absolute' height of the fracture relief $z=f(x, y)$

$$
H_{a}=\frac{1}{L \cdot M} \iint_{(L M)}|f(x, y)| \mathrm{d} x \mathrm{~d} y
$$

where $L \times M$ is the area of the vertical projection of the $3 \mathrm{D}$ fracture profile $f(x, y)$ into the plane $\mathrm{xy}$. Parameter $H_{a}$ has great statistical relevancy, since it is a global averaged characteristic covering the entire tested surface $L \times M$. The $3 \mathrm{D}$ profiles $f(x, y)$ were created using the Olympus Lext 3100 confocal microscope. One of these profiles is shown in Figure 1. The profiles are formed by the software that processed a series of optical sections created by the confocal microscope at various heights of the fracture surfaces. Approximately 150 image sections were taken for each measured surface site, starting from the very bottom of the surface depressions (valleys) and proceeding to the very top of the surface protrusions (peaks). The investigated area $L \times M=$ $1280 \mu \mathrm{m} \times 1280 \mu \mathrm{m}(1024$ pixels $\times 1024$ pixels $)$ was 


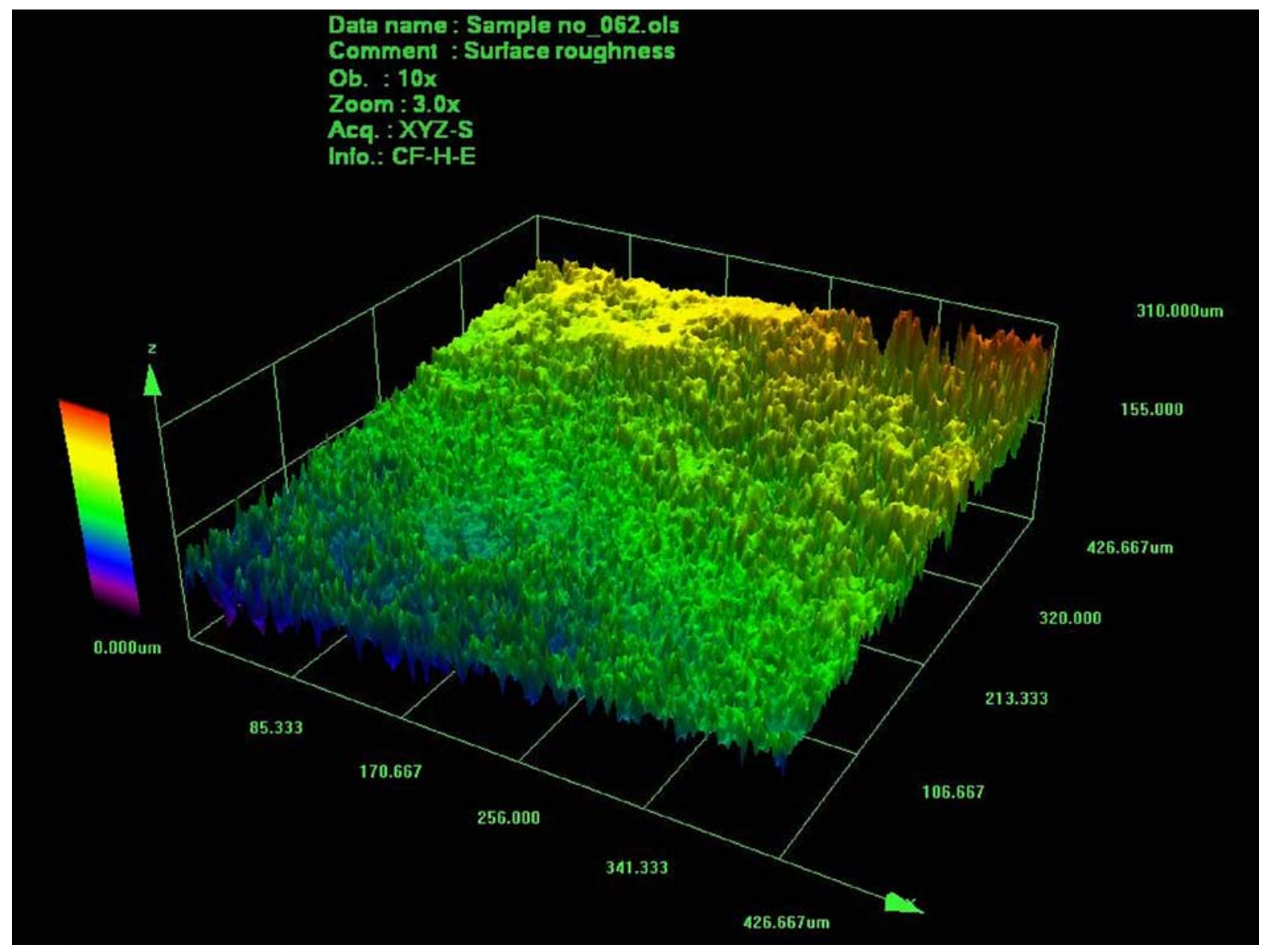

Fig. 1: 3D confocal relief of fractured cement paste

chosen in five different places of each fracture surface (in the center, and in four positions near the corners of the rectangular area), i.e. each plotted point on the graphs of the profile parameters corresponds to an average value composed of 90 measurements (18 samples $\times 5$ surface measurements). Each measurement was performed for three different magnifications, namely $5 \times, 20 \times$ and $50 \times$, giving 270 measurements that were performed for the particular $r$-value. Since each site measurement amounts to about 150 optical sections (digital files), 40500 files had to be processed to create 270 digital maps per one $r$-value. This resulted in 1620 digital maps for all $r$-values altogether $(6 r$-values $\times 270$ maps for each $r$-value) . These 1620 digital fracture surfaces were then subjected to $3 \mathrm{D}$ profile surface analysis.

In this way an extensive statistical ensemble was created to provide a sufficiently reliable basis for drawing relevant conclusions.

\section{Results and discussion}

Figure 2 presents three dependences of $H_{a}(P)$ formed within three magnifications: $5 \times, 20 \times$ and $50 \times$. Their graphs do not manifest linear behavior. All the three graphs show very similar shapes, which are well described by non-linear functions (curves in Figure 2) that can be expressed as follows

$$
H_{a}(P)=\frac{H_{o}}{\left(P_{o}-P\right)^{\beta}}+h_{o}
$$

This is in fact a power-law function pointing to fractal-like properties. Relation (2) contains four positive fitting parameters $H_{o}, P_{o}, \beta$, and $h_{o}$, the meanings of which can be explained on the basis of asymptotic patterns. Firstly, $P_{o}$ must always be greater than variable $P$, otherwise function (2) would be a decreasing function, and this would contradict the experimental data in Figure 2. This means that $P_{o}$ is the limiting value of porosity $P$ when the waterto-cement ratio $r$ goes to infinity

$$
P_{o}=\lim _{r \rightarrow \infty} P=1
$$

Assuming $P_{o}=1$ and $P \rightarrow 0$ (material with 'zero porosity'), the limiting roughness of the corresponding fracture surfaces reads

$$
\begin{aligned}
& \lim _{P \rightarrow 0} H_{a}(P)=H_{o}+h_{o} \\
& P_{o}=1
\end{aligned}
$$



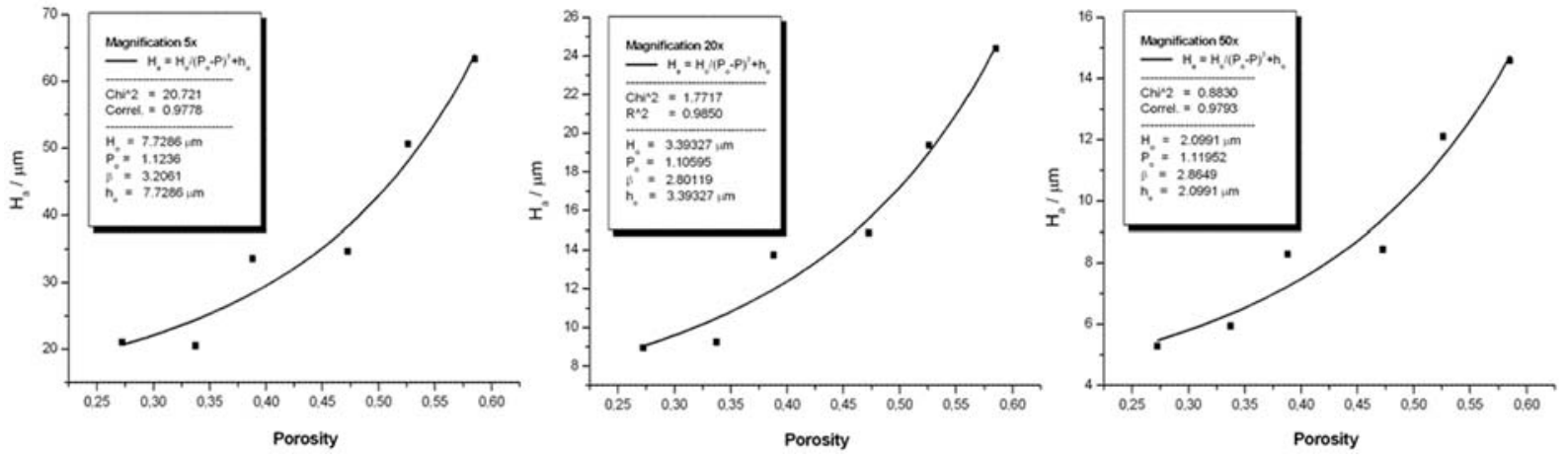

Fig. 2: The dependence $H_{a}(P)$ between the roughness of the fracture surfaces and the porosity of the cement paste
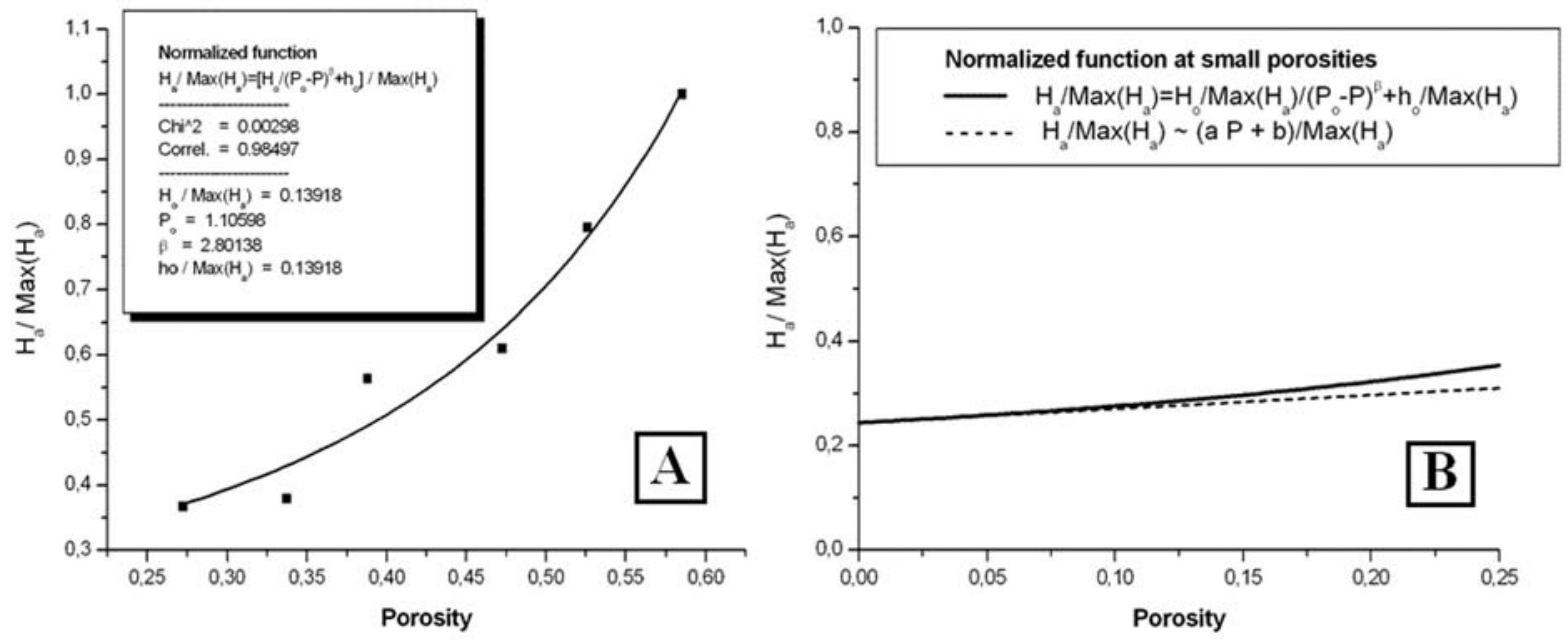

Fig. 3: Normalized scale-invariant data $H_{a} / \operatorname{Max}\left(H_{a}\right)$ in dependence on porosity $P$

From (4) it follows that the sum $H_{o}+h_{o}$ represents the height irregularity at 'zero porosity'. Since different magnifications provide different resolutions, 'zero porosity' will be determined differently for different magnifications, and thus the sum $H_{o}+h_{o}$ will also vary with magnification. The asymptotic form of $H_{a}(P)$ when the material contains a very small but non-zero porosity value, e.g. $0<P<0.30$ can also be useful for further consideration. In this case, the Taylor expansion of (2) may be utilized

$$
\begin{gathered}
H_{a}(P) \approx \frac{H_{o}}{P_{o}^{\beta}}\left(1+\beta \frac{P}{P_{o}}\right)+h_{o}=a P+b \\
a=\frac{\beta H_{o}}{P_{0}^{1+\beta}}, \quad b=\frac{H_{o}}{P_{o}^{\beta}}+h_{o} .
\end{gathered}
$$

It is obvious from (5) that at sufficiently small porosities the roughness of the fracture surfaces can be approximated by a linear function $H_{a}(P) \approx a P+b$, which is in agreement with the observation of Ponson [10], whose experiments with glass ceramics $(P<$ 0.3 ) indicated such behavior (figure 3.3 in Ref [10]).

Our data in Figure 2 does not contain a sufficient number of experimental points in the region of small porosities $(P<0.35)$, within which linear behavior can be observed. However, the clearly nonlinear behavior of function $H_{a}(P)$ at higher porosities is a real fact illustrated by all the graphs in Figure 2. The interval $P \in(0.35,0.50)$ is characterized by an abrupt non-linear increase, and at $P>0.50$ rapid growth unmistakably continues further. With our specimens, these three intervals of porosities $P<0.35, P \in(0.35,0.50)$ and $P>0.50$ correspond to the following water-to-cement ratios: $r_{1}<0.4, r_{2} \in(0.4,0.6)$ and $r_{3}>0.6$, respectively. It is well known that at sufficiently small water-to-cement ratio values $\left(r_{1}<0.4\right)$, gel porosity dominates over capillary porosity in hydrated cement pastes. Within the specimens mixed with intermediate $r$-values $r_{2} \in(0.4,0.6)$, capillary porosity starts to assume a governing role, and at still higher $r$-values $r_{3}>0.6$ the porosity of the specimens is prevalently formed by capillary porosity. The overall non-linear surface irregularity (2) of cementitious materials seems to be a result of the interplay between gel and capillary porosities. As soon as the onset of capillary porosity appears, the height irregularities of the fracture surfaces increase their values abruptly, as can be seen with all the graphs in Figure 2 and still more straightforwardly with the two graphs in Figure 3, where the boundary between the 
gel and the capillary porosities can be recognized at $P \approx 0.3$. This effect can be characterized as a crossover to larger scale porosities.

It is interesting to note that the shapes of the graphs of functions $H_{a}(P)$ shown in Figure 2 are mutually very similar, regardless of the magnifications that are used. In this connection, the question of their scale invariance arises. This means that the dependence $H_{a}(P)$ can be described by the same functional type (2), but with the fitting parameters $H_{O}$, $P_{o}, \beta, h_{o}$ adapted to the results of the particular magnification. However, when these three functions are normalized by using the highest measured values $\operatorname{Max}\left(H_{a}\right)$, a unified function results (Figure 3A). Using the pattern of the unified function, the linear behavior of surface roughness at small porosities can be illustrated straightforwardly — see Figure 3B.

The graph of the unified function shown in Figure $3 \mathrm{~A}$ represents the results of all three magnifications used here $-5 \times, 20 \times$ and $50 \times-$ and it simultaneously manifests the scale invariant properties of the height irregularities of the fracture surfaces.

\section{Conclusion}

The experiments have proved a close relationship between the roughness (height irregularities) of fracture surfaces and the porosity of materials. The functional relation of these two quantities is generally non-linear and may be described by a power law function (2). When normalizing this function, it becomes independent of the magnification that is used. The non-linear behavior of $H_{a}(P)$ with cement pastes is the result of the influence of gel and capillary porosities. The region of small gel porosities is characterized by a moderate (almost linear) increase in surface roughness $H_{a}(P)$, whereas the region of capillary porosity is a domain with an abrupt increase in this quantity.

The presented properties of the surface roughness of fractured cement pastes may also be useful for morphological and structural studies of other porous materials.

\section{Acknowledgement}

This work was supported by the Ministry of the Czech Republic under Contract no. ME09046 (Kontakt).

\section{References}

[1] Lange, D. A., Jennings, H. M., Shah, S. P.: A fractal approach to understanding cement paste microstructure, Ceram. Trans. 16 (1992) $347-363$
[2] Issa, M. A., Hammad, A. M.: Fractal characterization of fracture surfaces in mortar, Cem. Concr. Res. 23 (1993) 7-12.

[3] Lange, D. A., Jennings, H. M., Shah, S. P.: Analysis of surface-roughness using confocal microscopy, J. Mater. Sci. 28 (14) (1993) $3879-3884$.

[4] Lange, D. A., Jennings, H. M., Shah, S. P.: Relationship between fracture surface roughness and fracture behavior of cement paste and mortar, J. Am. Ceram. Soc. 76 (3) (1993) 589-597.

[5] Zampini, D., Jennings, H. M., Shah, S. P.: Characterization of the paste-aggregate interfacial transition zone surface-roughness and its relationship to the fracture-toughness of concrete, J. Mater. Sci. 30 (12) (1995) 3149-3 154.

[6] Lange, D. A., Quyang, C., Shah, S. P.: Behavior of cement-based matrices reinforced by randomly dispersed microfibers, Adv. Cem. Bas. Mater. 3 (1) (1996) 20-30.

[7] Abell, A. B., Lange, D. A.: Fracture mechanics modeling using images of fracture surfaces, Int. J. Solids Structures, 35 (31-32) (1997) $4025-4034$.

[8] Nichols, A. B., Lange, D. A.: 3D surface image analysis for fracture modeling of cementbased materials, Cem. Conc. Res. 36 (2006) 1 098-1 107.

[9] Ficker, T., Martišek, D., Jennings, H. M.: Roughness of fracture surfaces and compressive strength of hydrated cement pastes, Cem. Conr. Res. 40 (2010) 947-955.

[10] Ponson, L.: Crack propagation in disordered Materials; How to decipher fracture surfaces, Annales de Physique 32 (2007) 1-120.

[11] Ponson, L., Auradou, H., Pessel, M., Lazarus, V., Hulin, J. P.: Failure mechanisms and surface roughness statistics of fractured Fontainebleau sandstone, Phys. Rev. E $\mathbf{7 6}$ (2007) 036108/1-036108/7.

Prof. RNDr. Tomáš Ficker, DrSc.

Phone: +420 541147661

E-mail: ficker.t@fce.vutbr.cz

Department of Physics

Faculty of Civil Engineering

Brno University of Technology

Veveří 95, 66237 Brno, Czech Republic 